\title{
The Pathology of Tumours at the Limbus
}

\author{
ALEC GARNER \\ London
}

\begin{abstract}
Summary
The histopathology of tumours originating at the corneoscleral limbus is reviewed with special reference to some 636 specimens examined over a 40 year period at the Institute of Ophthalmology, London. Over half the lesions involved melanocytes (351 cases) derived from the limbal conjunctiva and of these two-thirds were benign naevi. Conversely, benign squamous cell tumours were rare, only five unequivocal papillomas having been seen compared with 137 premalignant lesions and 73 carcinomas. Solid dermoids accounted for approximately 10 per cent of all tumours involving the limbus.
\end{abstract}

The concept of a corneoscleral limbus has value as an anatomical landmark but by definition has no separate histological identity. Correspondingly, from a histogenetic point of view there is no such thing as a limbal tumour and the tumours that are recognised in this region reflect the various tissue types that are to be found there.

In practice the vast majority of tumours at the limbus arise from the conjunctival epithelium, the remainder involving the connective tissues and virtually none originating from the corneal margin. Over a forty year period at the Institute of Ophthalmology, London, we have examined some 636 tumours involving the limbus, distributed as follows:

Congenital lesions: Dermoid 68

Melanocytic naevus 214

Benign Neoplasms: Squamous papilloma 5

Fibroma 2

Precancerous lesions: Epithelial dysplasia 30

Carcinoma-in-situ 107

Acquired melanosis 12
Malignant neoplasms: Squamous carcinoma 73

Melanoma 125

\section{Congenital Lesions \\ Dermoid}

The limbus, most commonly in the inferotemporal region, is a preferred site for this hamartomatous lesion. Presumed to be due to the sequestration of epidermal and related connective tissue elements during embryogenesis, dermoids are composed of a fairly discrete mass of fibrous tissue embedded in which occasional hair-follicles, sebaceous gland acini and, rarely, sweat gland structures may be seen (Fig. 1). Almost all limbal dermoids are solid, only one of the specimens in the present review being cystic. Adipose tissue may also be a feature and when prominent the designation dermolipoma is applicable and, although dermolipomas in general tend to predominate near the lateral canthus, 21 of the 68 limbal dermoids in the present series included unmistakable adipose tissue.

Clinically dermoids are generally well defined with a smooth surface and may have a

From Department of Pathology, Institute of Ophthalmology, University of London.

Correspondence to: Professor A. Garner, 17/25 Cayton Street, London EC1V 9AT. 


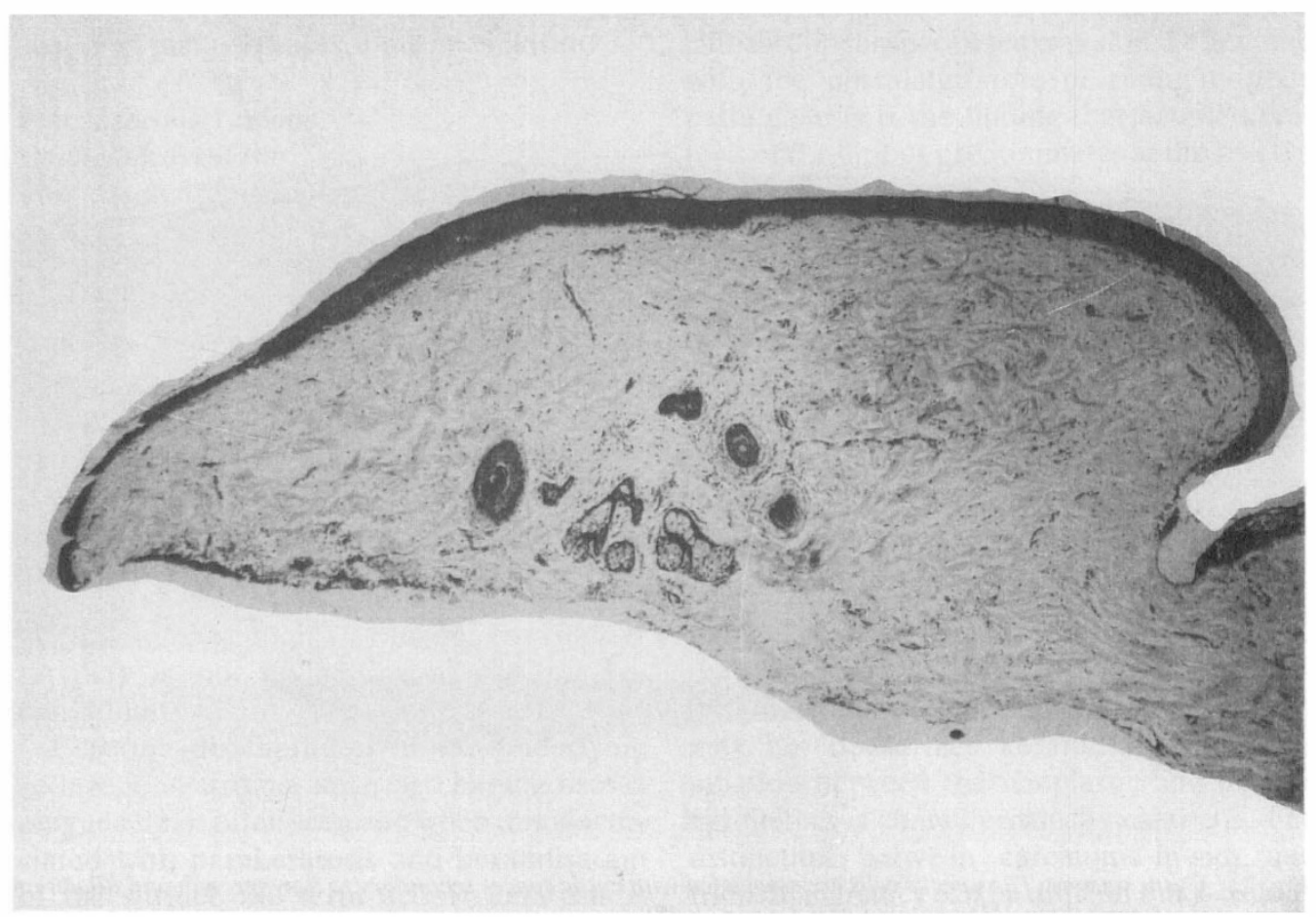

Fig. 1. Limbal dermoid showing hair-follicles and sebaceous gland acini within a discrete, elevated nodule of fibrous connective tissue (Haematoxylin and eosin, $\times 45$ ).

yellowish appearance. While presentation during infancy is to be expected, 54 per cent of our cases presenting within the first five years of life, manifestation may be delayed and we have received specimens from patients as old as 65 years. They may recur following local excision since it is not always easy to identify the base of the lesion and corneal scarring can also ensue. Occasionally they encircle the limbus and are bilateral, when they are likely to be dominantly inherited ${ }^{1}$ or they may be seen as part of Goldenhar's oculoauriculovertebral syndrome.?

\section{Melanocytic naevi}

Naevi developing at the limbus are essentially conjunctival lesions, the cornea normally being devoid of melanocytes, and, apart from rare stromal melanocytic proliferations (blue naevi), involve cells which, like the epithelial melanocytes, have originated from the neural crest.

Limbal naevi correspond with those of the conjunctiva in general but, particularly where there is extension onto the cornea, they tend to be slightly raised lesions because of the resistance to downward spread provided by the compact corneoscleral envelope. They vary considerably in the degree of pigmentation and amelanotic naevi are quite common. Of the 153 cases for which the information was available 61 (39.9 per cent) presented before the age of 15 years and 107 (69.9 per cent) occurred within the first 30 years of life. Nevertheless we have seen one example in a patient in her 98th year!

Histologically they resemble conjunctival naevi in every way, including a propensity for cystic change (Fig. 2). The capacity to form cystic inclusions is most often seen in lesions showing junctional activity and represents the downgrowth of epithelium from the surface. The downgrowths frequently include goblet cells and, should the neck of the inclusion become obstructed, the continued secretion of mucus can result in cystic distension suffi- 


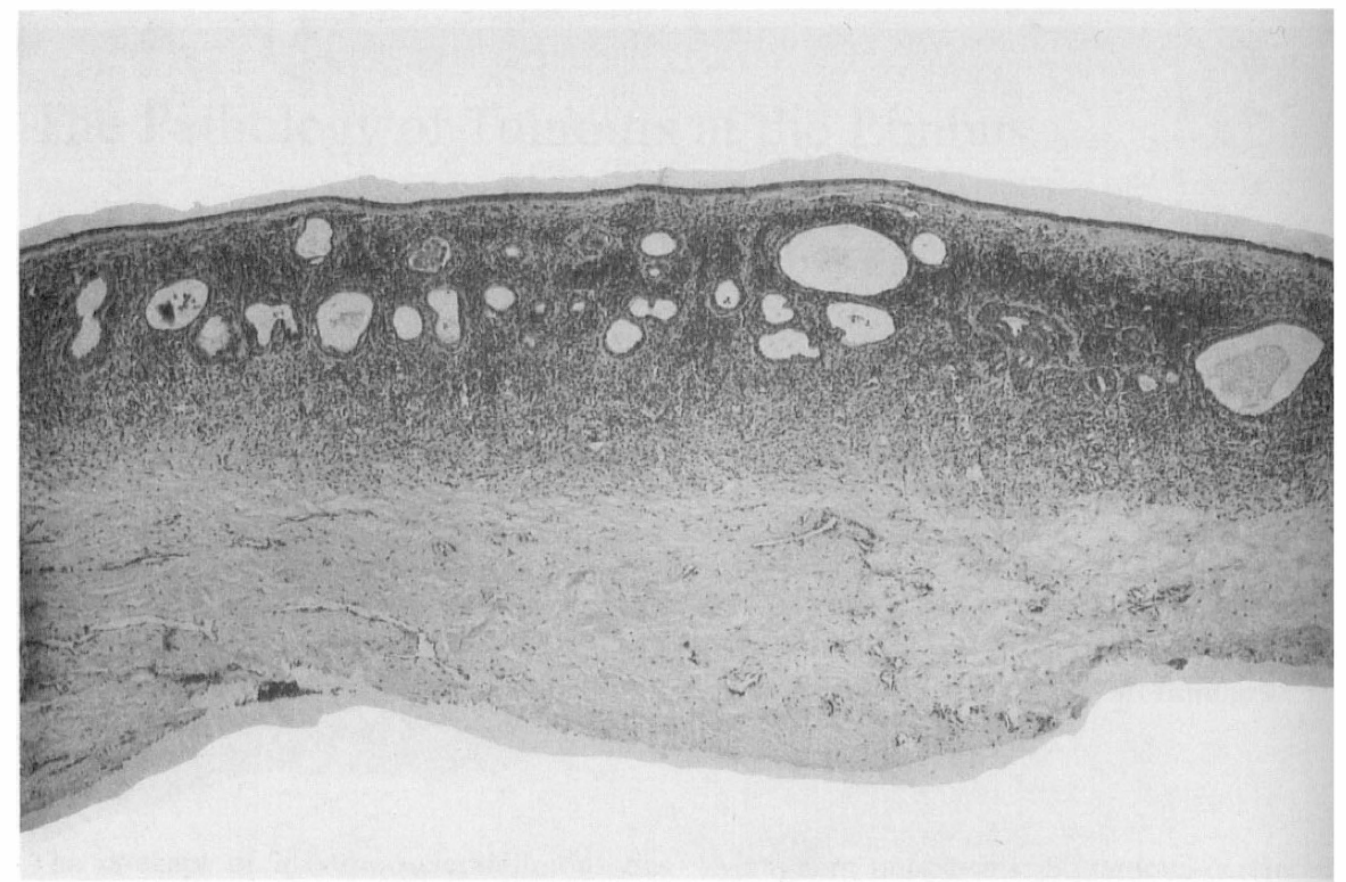

Fig. 2. Cystic naevus. The naevus cells are associated with cystic spaces secondary to downgrowth and dilatation of the surface epithelium (Haematoxylin and eosin, $\times 45$ ).

cient on occasion to arouse fears of malignant change. In practice the prognosis for cystic naevi is exactly the same as that for other conjunctival naevi. We have seen one example of a blue naevus attributable to proliferation of spindle-shaped stromal melanocytes.

Very occasionally naevi are encountered in which the cells include spindle-shaped and epithelioid forms as well as or instead of the usual innocuous cell types. Both intraepithelial and stromal activity is seen and the appearance can be suggestive of malignancy so that the term juvenile melanoma is commonly applied but lesions of this sort occurring, as they almost always do, in children or young adolescents are invariably benign. We have observed two such cases.

\section{Benign Neoplasms}

\section{Conjunctival papilloma}

The characteristic histological feature of a conjunctival papilloma is the proliferation of regular epithelium to form fronds or fingerlike processes projecting from the surface.
However, those presenting at the limbus are often relatively flat with a sessile base such that the papillomatous nature of the lesion may not be too obvious. The epithelium is usually non-keratinising although mucussecreting goblet cells are sparse. In our experience this is an uncommon tumour at the limbus and some ostensible cases on our files have had to be rediagnosed on review as welldifferentiated epithelial dysplasias or squamous carcinomas. Limbal papillomas, like the conjunctival lesion in general, can occur at any age but most do so in middle age and rarely recur following local removal.

\section{Fibroma}

Consisting of a plaque or nodule of hyalinised collagenous tissue attached to the surface of the corneoscleral stroma in the absence of a defined capsule, the diagnosis of fibroma is rather nebulous. Comparison with the fibrous component of a solid dermoid is certainly justified and diagnosis must be based on multiple sections to exclude the presence of sparsely 
distributed epidermal structures. We have observed just two cases, both in children.

\section{Precancerous Lesions \\ Epithelial dysplasia}

The affected epithelium is slightly thickened since the dysplasia is expressed in terms of increased cell proliferation as well as irregularity of the individual cells. The cellular abnormality is concentrated in the deeper layers of the epithelium in the form of increased nuclear size, nuclear hyperchromatism, variable cell size and shape, and some loss of cell polarity (Fig. 3). These changes may vary between cases from mild to severe. Goblet cells are absent in the affected area and there is sometimes a degree of parakeratosis, although cellular irregularity (atypia) is not a feature of the surface epithelium.

Elastotic degeneration of the underlying collagenous stroma, such as characterises a pinguecula, is often seen and when this is associated with parakeratosis and keratinisation of the surface the term actinic keratosis is applicable. Such lesions correspond with a clinical appearance of leukoplakia. In keeping with the postulated role of sunlight in its pathogenesis is the finding that actinic keratosis of the limbus predominates at the level of the palpebral fissure.

\section{Carcinoma-in-situ}

The clinical appearance is variable but commonly carcinoma-in-situ of the conjunctival epithelium presents as a circumscribed, raised area of translucent milky-white tissue. As a rule the surface is smooth although occasionally it can simulate a papilloma (our files include seven specimens initially diagnosed on clinical appearances as squamous papilloma). Histologically it differs from epithelial dysplasia only in the extent of the derangement, the cellular atypia involving the full thickness of the epithelium. There may or may not be surface keratinisation but the junction between the neoplastic and normal epithelium is characteristically clear cut. The distinction between carcinoma-in-situ and frank malignancy can be difficult but is usually

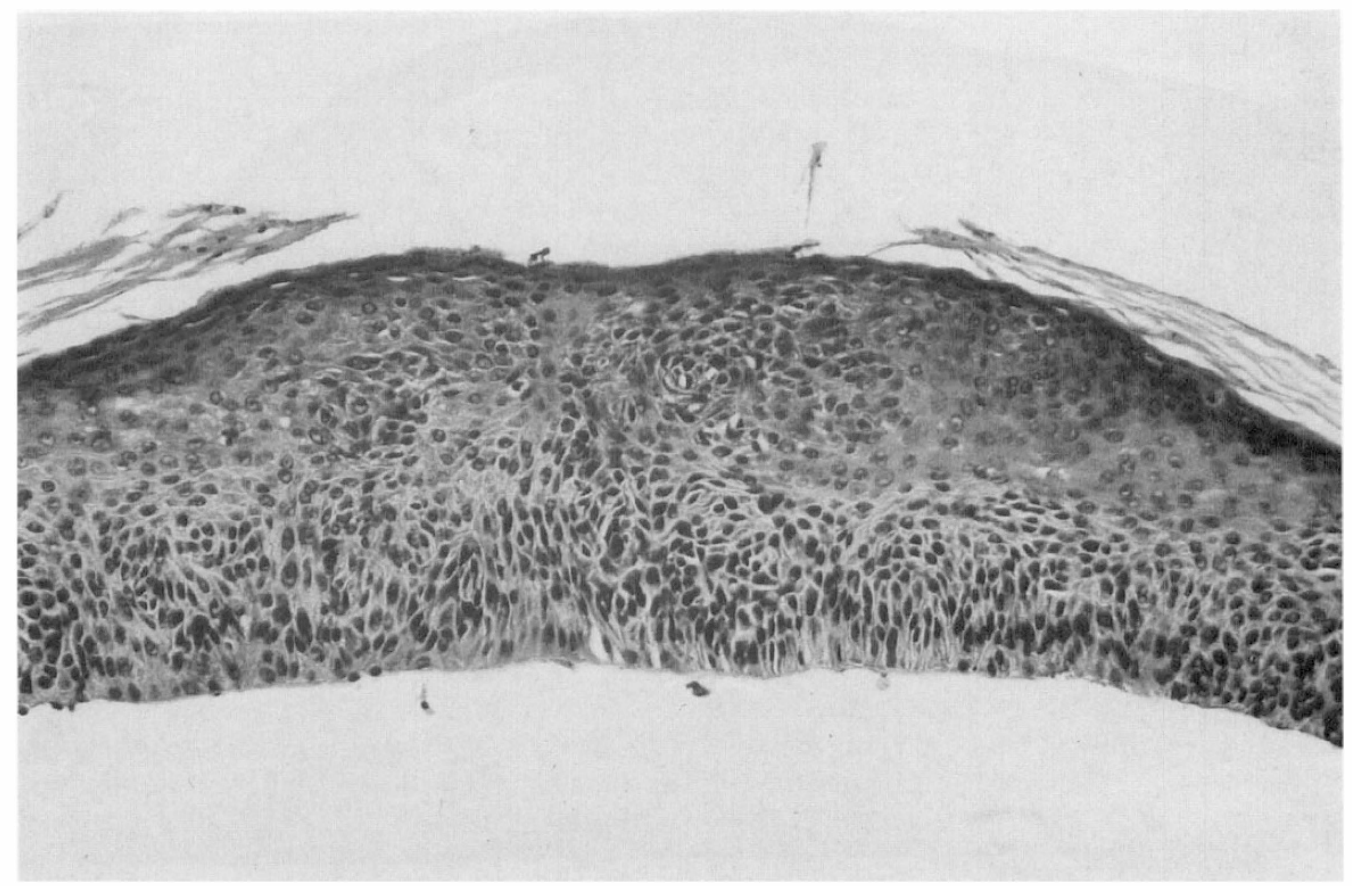

Fig. 3. Dysplasia of the limbal epithelium. The abnormality is largely confined to the deeper layers of the epithelium, where the cells are pleomorphic with hyperchromatic nuclei and some loss of polarity (Haematoxylin and eosin, $\times 180)$. 
made on the basis of an intact basement membrane.

Untreated, gradual spread onto the cornea is to be expected and there is a small risk that an invasive lesion will supervene, one study giving an incidence of about 3 per cent. ${ }^{3}$ The same investigators reported a recurrence rate of 24 per cent following local excision. In passing it might be noted that the frequent appellation, Bowen's disease, to this disorder is not strictly applicable since the original description of this condition referred to a skin lesion characterised by a scaly eruption simulating chronic eczema or psoriasis.

\section{Acquired melanosis}

A spontaneous localised increase in melanocytes within the conjunctival epithelium which is not present at birth is known as primary acquired melanosis. The affected area is often ill-defined but the bulbar conjunctiva near to the limbus is a favoured site. Most cases develop in adult life and the lesions may be observed to wax and wane as judged by the degree of pigmentation. The risk that an invasive melanoma will occur varies con- siderably and is best assessed on the basis of biopsy examination using the classification introduced by Folberg et al. ${ }^{4}$

Primary acquired melanosis without atypia refers to an increased number of small polyhedral melanocytes, with scanty cytoplasm and bland nuclei with inconspicuous nucleoli, at the level of the basal layer of epithelium. In some instances the melanocytes may form clusters pushing up into the epithelium without invading it and preserving their basal attachment. With a median follow-up period of 13 years an analysis by Folberg and colleagues $^{4}$ revealed recurrence after local excision in one of 13 cases only and no instances of melanoma development. Some melanin pigment is released into the underlying stroma where it is engulfed by macrophages and, although this does not indicate invasive behaviour, it probably accounts for the spontaneous changes in size of the lesion sometimes seen at successive examinations.

Primary acquired melanosis with atypia is characterised by the migration of melanocytes into the more superficial layers of the epithelium, whilst the cells themselves are larger

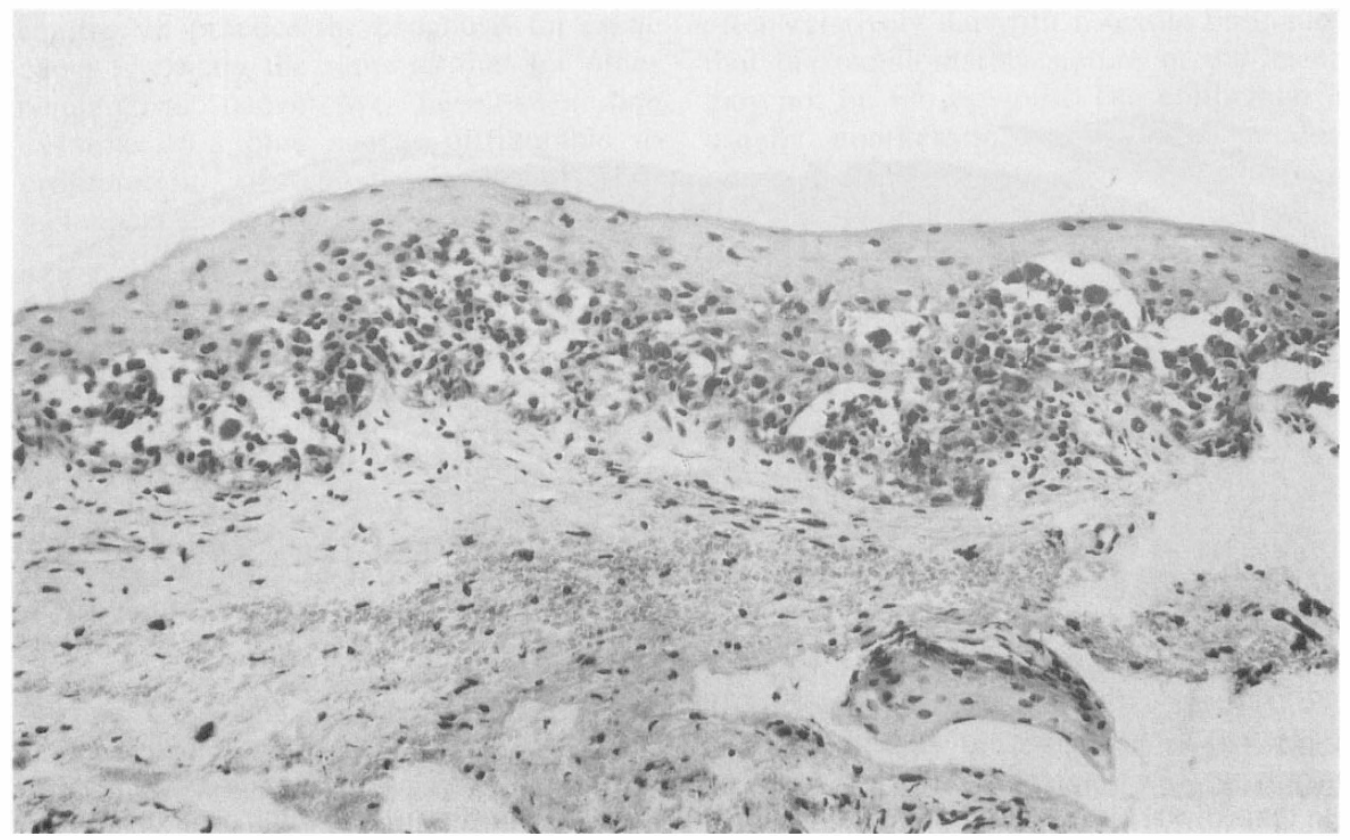

Fig. 4. Primary acquired melanosis with atypia. Irregularly shaped and sized melanocytes have moved away from the basal layer and are invading the more superficial epithelium (Haematoxylin and eosin, $\times 180)$. 
with increased amounts of cytoplasm, enlarged nuclei and prominent nucleoli (Fig. 4). Lesions of this sort have a much worse prognosis, 13 of 28 cases investigated in the study by Folberg and his colleagues ${ }^{4}$ progressing to malignant melanoma.

\section{Malignant Neoplasms}

Squamous carcinoma

Squamous cell carcinoma of the conjunctiva may arise de novo or be preceded by a recognised in situ phase and predominates at the limbus where it usually presents as a papillary tumour projecting from the surface.

In the majority of cases the tumour is well differentiated, the cells exhibiting clearly identifiable intercellular junctions and a tendency to be arranged in nests at the centre of which keratin 'pearls' may be seen. Occasionally, however, more anaplastic forms are encountered. Extension onto the cornea, if it occurs, is usually anterior to Bowman's layer although invasion of the substantia propria can occur secondary to direct penetration of the sclera at the limbus (Fig. 5). Invasion of the anterior chamber is rare save in untreated cases, each of the three instances we have seen being in patients from the third world for whom treatment facilities were inadequate. Retrograde spread into the orbit may also supervene in neglected cases but metastasis via the lymphatic drainage channels to the preauricular or submandibular lymph nodes is very rare. In one study involving 87 cases followed up for a minimum period of five years metastasis occurred in only four patients. ${ }^{5}$ The prognosis in the event of prompt surgical excision is usually excellent.

\section{Malignant melanoma}

The limbus is also a preferred location for conjunctival melanoma to develop in the form

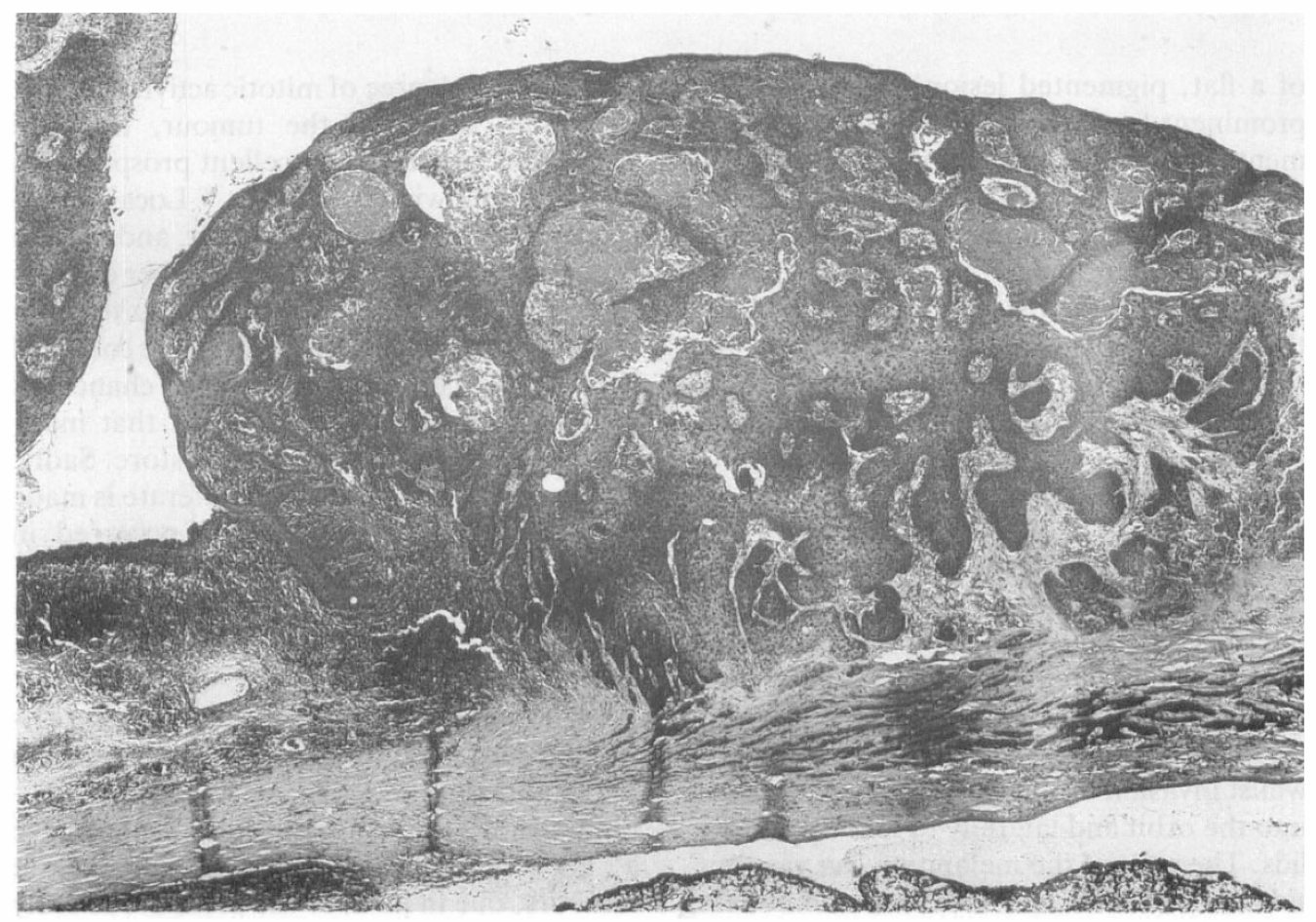

Fig. 5. Squamous cell carcinoma originating at the limbus with invasion of the cornea in a pattern determined by the lamellar organisation of the stroma. There is intense inflammation in the perilimbal conjunctiva (Haematoxylin and eosin, $\times 30)$. 


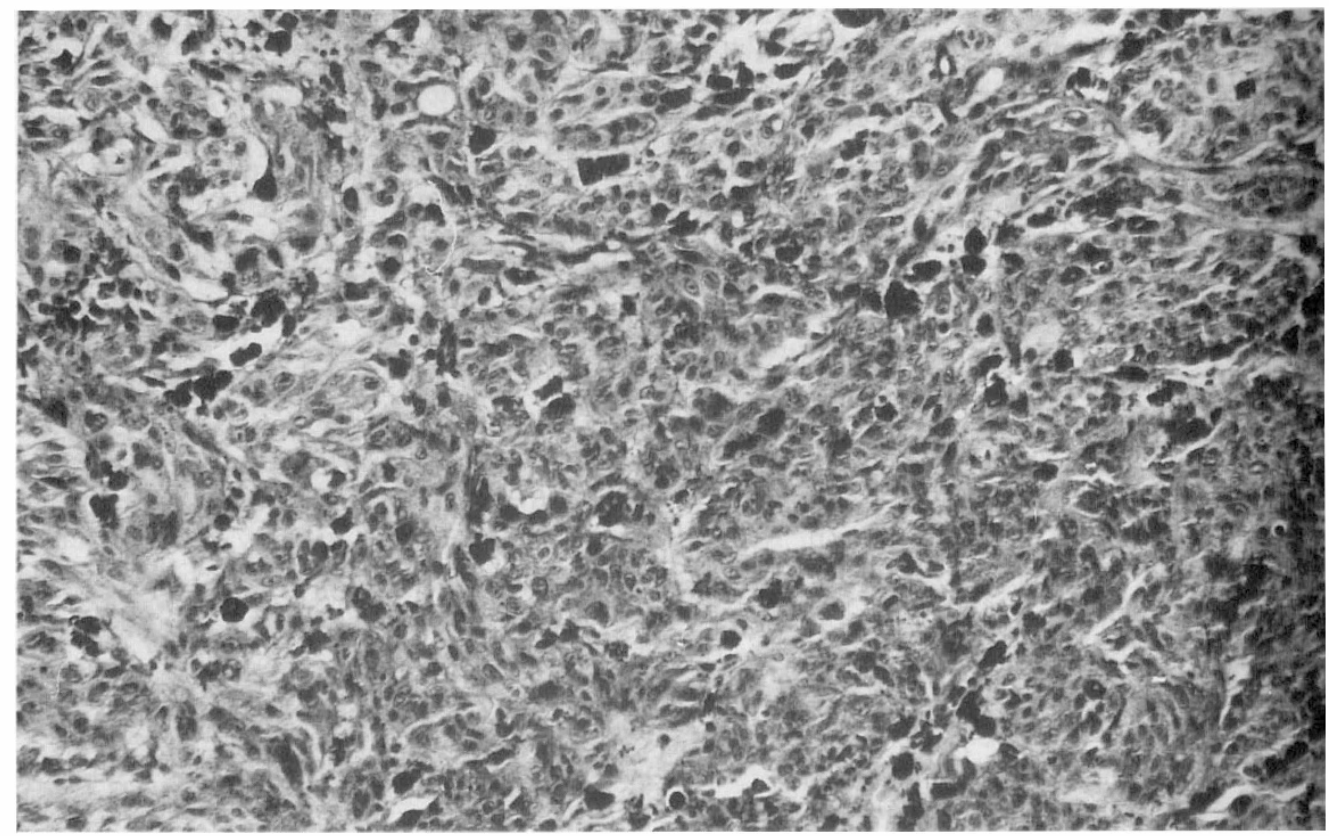

Fig. 6. Malignant melanoma of the limbal conjunctiva showing scattered melanin deposits and pleomorphic cells with prominent nucleoli (Haematoxylin and eosin, $\times 180)$.

of a flat, pigmented lesion which may have prominent feeder vessels. The degree of pigmentation can vary quite considerably but we have only one truly amelanotic limbal melanoma on file. Origin from a preceding naevus or primary acquired melanosis accounts for about three quarters of the cases, the former being said to have a better prognosis than the latter ${ }^{6}$ although a more recent analysis casts doubt on this claim. ${ }^{7}$ The remainder appear to arise de novo. Tumours presenting in patients over 70 years age are also claimed to be less aggressive. ${ }^{8}$

Histologically the tumours may involve both the deep tissues and the surface epithelium. The epithelial component is associated with destruction and even ulceration of this layer with, in some instances, Pagetoid spread through the marginal epithelium, whilst invasion of the stroma can extend back into the orbit and laterally to involve the eyelids. The cells of the melanoma can vary considerably from case to case, some being epithelioid with pleomorphic nuclei and conspicuous nucleoli whereas others show far less atypia (Fig. 6). Prognosis seems to correlate best with the degree of mitotic activity and the maximum depth of the tumour, those of $1.5 \mathrm{~mm}$ or less having excellent prospects for survival following treatment. ${ }^{9}$ Local recurrence is not infrequent, however, and may be repeated several times over a number of years and herein lies the dilemma: whether to exenterate the orbit relatively early in the course of the disease in order to improve the chances of survival or to delay in the hope that many more years of useful vision are in store. Sadly, by the time the decision to exenterate is made distant metastasis has already occurred in many cases.

\section{Conclusion}

As will be appreciated from the foregoing survey the corneoscleral limbus is a common site for tumours of the outer eye to develop. Fortunately the vast majority of such lesions have a good prognosis, even the malignant tumours, due in part, to the prospect of early detection and the limited capacity for spread given the rigid and largely impenetrable nature of the corneoscleral envelope. 


\section{References}

${ }^{1}$ Mattos J, Contreras F, O'Donnell FE Jr: Ring dermoid syndrome: a new syndrome of autosomal dominantly inherited, bilateral, annular limbal dermoids with corneal and conjunctival extension. Arch Ophthalmol 1980, 98: 1059-61.

2 Sugar HS: The oculoauriculovertebral syndrome of Goldenhar. Am J Ophthalmol 1966, 62: 678-82.

${ }^{3}$ Erie JC, Campbell RJ, Liesegang TJ: Conjunctival and corneal intraepithelial and invasive neoplasia. Ophthalmol 1986, 93: 176-83.

${ }^{4}$ Folberg R, McLean IW, Zimmerman LE: Primary acquired melanosis of the conjunctiva. Hum Pathol 1985, 16: 129-35.

${ }^{5}$ Zimmerman LE: The cancerous, precancerous and Pseudocancerous lesions of the cornea and con- junctiva. In Rycroft PV ed. Corneo-Plastic Surgery. Oxford: Pergamon, 1969: 547-555.

${ }^{6}$ Reese AB: Precancerous and cancerous melanosis. Am J Ophthalmol 1966, 61: 1272-7.

${ }^{7}$ Folberg R, McLean IW, Zimmerman LE: Malignant melanoma of the conjunctiva. Hum Pathol 1985, 16: $136-43$.

${ }^{8}$ Allen AC and Spitz S: Malignant melanoma; a clinicopathological analysis of the criteria for diagnosis and prognosis. Cancer 1953, 6: 1-45.

${ }^{9}$ Silvers DN, Jakobiec FA, Freeman TF, Lefkowitch JH, Ellie RC: Melanoma of the conjunctiva: a clinicopathologic study. In Jakobiec FA ed. Ocular and Adnexal Tumors. Birmingham, Ala: Aesculapius 1978: 583-99. 